\title{
Les accidents d'irradiation, 1950-2000 leçons du passé
}

\author{
J.-C. NÉNOT ${ }^{1}$
}

(Manuscrit reçu le 30 mai 2001, accepté le 16 juillet 2001)

RÉSUMÉ Les accidents causés par les rayonnements ionisants sont rares et possèdent leur propre spécificité. Ils ont des effets indirects, particulièrement importants dans les accidents de grande ampleur. La gamme des accidents est large et variée : il existe des accidents qui ne posent pas de problèmes particuliers, des accidents dont la gestion médicale est délicate et des accidents qui mobilisent des moyens très importants. Dans chacune de ces classes on peut distinguer les accidents qui sont diagnostiqués d'emblée et ceux qui sont découverts tardivement et qui auraient pu être ignorés. Certains accidents sont gardés secrets pour des raisons militaires ou politiques. Cette classification permet de tirer un enseignement général, qui devrait contribuer à la prévention et à la gestion des situations accidentelles, tant en ce qui concerne la sûreté que le domaine médical.

\begin{abstract}
Radiation accidents: historical review 1950-2000
Accidents caused by ionising radiations are rare and their direct health consequences are specific. The indirect effects of large-scale accidents are particularly important. The range of radiation accidents is wide and varied: some accidents do not raise any particular question, while the management of some others is tricky, either because of medical problems or because of the large size of the accident. In each of these categories, accidents that are immediately diagnosed should be distinguished from those that are recognised only after a certain delay and might have been completely ignored. Another category includes the accidents that are kept secret for military or political reasons. Lessons may be derived from such a classification, which should contribute to the prevention and management of accidental situations, with regards to safety and medical handling.
\end{abstract}

\section{Introduction}

Par définition, un accident est un événement fortuit, non prévisible, qui entraîne ou risque d'entraîner des dommages pour la santé de l'homme. Dans le contexte des accidents causés par les rayonnements, cette définition demande à être précisée.

- Les accidents qui mettent en cause les rayonnements ionisants sont des événements très rares. Chaque accident constitue un événement unique, et il ne faut pas espérer pouvoir tirer d'un seul accident des règles génériques pour la prévention ou la gestion des futurs accidents.

1 Institut de protection et de sureté nucléaire, BP 6, 92265 Fontcnay-aux-Roses Cedex, France. 
- Les dommages qu'ils causent présentent des caractères particuliers qui peuvent compliquer la tâche des spécialistes impliqués. Par exemple, la période de latence de certaines réactions peut inciter à un optimisme injustifié et entraîner des décisions erronées.

- La définition d'accident s'appuie en général sur l'existence de dommages médicaux apparents. C'est vrai quand les rayonnements induisent des effets aigus. C'est beaucoup plus difficile de trancher en l'absence d'effets aigus ; néanmoins il peut exister un risque inacceptable d'induction de cancers.

- Suivant la nature de l'accident, l'accidenté peut souffrir d'affections variées, qui ne sollicitent pas les mêmes spécialistes : une irradiation aiguë globale requiert une réanimation hématologique, une brûlure radiologique peut nécessiter des actes de chirurgie fine et une contamination interne fait appel à des traitements spécifiques.

- Il existe des effets indirects, particulièrement importants dans les accidents de grande ampleur et qui peuvent concerner des individus qui n'ont pas subi l'exposition accidentelle.

- Il existe une gamme d'accidents radiologiques et nucléaires extrêmement large et variée. Dans le cas le plus simple, l'accident ne cause qu'une seule victime, la source est connue et identifiée, le diagnostic de l'accident et celui de la maladie sont faciles; la déontologie habituelle règle alors normalement l'acte médical. La situation est souvent plus compliquée : victimes en grand nombre, lésions combinées rendant difficile le choix de la structure d'accueil la mieux adaptée, diagnostic malaisé, pronostic incertain et choix thérapeutique difficile ou controversé ; dans ce cas, les décisions d'ordre sanitaire reviennent aux pouvoirs publics.

Ces considérations générales permettent d'identifier deux critères principaux de classement :

(i) la façon dont les accidents sont découverts,

(ii) les difficultés de leur gestion, y inclus les problèmes médicaux et/ou sanitaires qu'ils posent.

Cette approche permet de distinguer les accidents dont le diagnostic est posé d'emblée de ceux qui ne sont découverts que tardivement. Les accidents gardés secrets pour des raisons militaires ou politiques se situent à part. Dans chaque groupe, il existe des accidents qui ne posent pas de problèmes particuliers, ceux dont la gestion médicale est délicate et ceux qui mobilisent des moyens très importants. Des exemples choisis pour illustrer cette classification figurent dans le tableau I, qui n'a aucun caractère exhaustif. Ce tableau appelle trois remarques d'ordre général :

- la fréquence des accidents graves ne semble pas diminuer avec le temps;

- les accidents concernent tous les pays et sont sans rapport avec le degré de développement économique ; 


\section{TABLEAU I}

Exemple de classification d'accidents en fonction de leurs difficultés de gestion (par pays et par année).

Classification of accidents, in terms of management difficulties (by country and year).

\begin{tabular}{|c|c|c|c|c|c|c|}
\hline \multirow[b]{2}{*}{$\begin{array}{l}\text { Accidents } \\
\text { reconnus d'emblée }\end{array}$} & \multicolumn{2}{|c|}{ Gestion simple } & \multicolumn{2}{|c|}{ Gestion délicate } & \multicolumn{2}{|c|}{ Catastrophes } \\
\hline & $\begin{array}{l}\text { Norvège } \\
\text { Israël } \\
\text { Belarus } \\
\text { Russie }\end{array}$ & $\begin{array}{l}1982 \\
1990 \\
1991 \\
1997\end{array}$ & $\begin{array}{l}\text { Pérou } \\
\text { Japon }\end{array}$ & $\begin{array}{l}1999 \\
1999\end{array}$ & Ukraine & 1986 \\
\hline $\begin{array}{l}\text { Accidents } \\
\text { méconnus }\end{array}$ & $\begin{array}{l}\text { Mexique } \\
\text { Italie } \\
\text { Algérie } \\
\text { Maroc } \\
\text { Salvador } \\
\text { Espagne } \\
\text { France } \\
\text { Vietnam } \\
\text { Egypte }\end{array}$ & $\begin{array}{l}1962 \\
1975 \\
1978 \\
1984 \\
1989 \\
1990 \\
1991 \\
1992 \\
2000\end{array}$ & $\begin{array}{l}\text { USA } \\
\text { UK } \\
\text { USA } \\
\text { USA } \\
\text { Chine } \\
\text { Estonie } \\
\text { Géorgie }\end{array}$ & $\begin{array}{l}1974-76 \\
1982-91 \\
1985-87 \\
1992 \\
1992 \\
1994 \\
1997\end{array}$ & $\begin{array}{l}\text { Mexique } \\
\text { Brésil } \\
\text { Costa Rica } \\
\text { Thaïlande } \\
\text { Panama }\end{array}$ & $\begin{array}{l}1983 \\
1987 \\
1996 \\
2000 \\
2001\end{array}$ \\
\hline Accidents secrets & & & URSS & 1961,68 & URSS & 1957 \\
\hline
\end{tabular}

- beaucoup d'accidents graves sont peut-être totalement inconnus, puisque le nombre d'accidents qui ont été découverts fortuitement est important et semble même augmenter avec le temps.

\section{Accidents reconnus d'emblée}

\subsection{Gestion simple}

Les points communs de ces accidents dont la gestion ne soulève pas de difficultés particulières sont: connaissance immédiate de l'accident, identification des victimes sans ambiguïté, lésions pouvant entrer dans le cadre de la médecine classique et logistique disponible adaptée. Cependant, des erreurs de toute sorte sont possibles, particulièrement en ce qui concerne le diagnostic et le traitement. Les exemples sont fournis par trois accidents dans des installations industrielles et par un accident de criticité dans une installation militaire, qui tous ont causé la mort d'un employé. Dans les trois premiers accidents, les employés ont reçu des doses largement en excès de 10 Gy suite à leur intrusion dans la salle d'irradiation avec la source de cobalt 60 en position d'irradiation (de quelques $\mathrm{PBq}$ à quelques dizaines de $\mathrm{PBq}$ ) ; le quatrième accident a été causé par une erreur humaine, rendue possible par un défaut de conception de l'ensemble expérimental destiné à étudier les réactions de fission, à des fins civiles et militaires. 
À Kjeller (Norvège, 2 septembre 1982), l'employé, dans le métier depuis une trentaine d'années, est mort en treize jours d'insuffisance rénale aiguë, sur un fond d'aplasie profonde (Stavem et al., 1995). L'évaluation des doses par analyse chromosomique n'a pas été possible en raison de dommages lymphocytaires considérables. L'évolution hématologique et l'examen par résonance paramagnétique électronique d'objets portés par la victime indiquent une dose moyenne d'environ $20 \mathrm{~Gy}$. À Soreq (Israël, 21 juin 1990), l'employé a violé deux consignes pour pénétrer dans la chambre d'irradiation. Deux heures après l'accident, la victime a été hospitalisée et sa dose évaluée entre 10 et $20 \mathrm{~Gy}$; six heures plus tard, un traitement anti-infectieux préventif a été combiné à un traitement par facteur de croissance hématopoiétique (GM-CSF) (IAEA, 1993). Une greffe de moelle a été pratiquée le quatrième jour avec la moelle du frère de la victime. Le patient est mort 36 jours après l'accident d'un syndrome digestif grave et de défaillance respiratoire. À Nesvizh (Belarus, 26 octobre 1991), la gravité de l'irradiation a été immédiatement reconnue et la victime transportée dans des délais très courts à Moscou (IAEA, 1996a). La dose a été évaluée supérieure à $10 \mathrm{~Gy}$, avec une répartition très inégale dans l'organisme. Les médecins russes refusèrent d'envisager une transplantation de moelle, car ils estimèrent que la restauration hématologique était possible et que la transplantation comportait plus de risques que d'avantages. Le patient, traité par facteurs de croissance (GM-CSF et IL-3), a restauré son hématopoïèse en quelques semaines. Cependant la détérioration de tous les autres systèmes fut rapidement dramatique; un syndrome digestif apparut en une semaine, suivi de brûlures étendues heureusement échelonnées dans le temps, de défaillances hépatique et rénale, compliquées par des problèmes pulmonaires, qui entraînent la mort du patient 113 jours après son irradiation. À Sarov (Russie, 17 juin 1997), dans le centre militaire d'Arzamas-16, des erreurs de manipulation sur un assemblage d'uranium causèrent une excursion critique ; l'employé fut exposé à un flux neutronique élevé, prépondérant par rapport à l'exposition gamma (IAEA, 2001). La dose moyenne fut estimée à environ $15 \mathrm{~Gy}$, avec un gradient très important suivant les régions du corps, certaines pouvant avoir reçu jusqu'à $60 \mathrm{~Gy}$. Moins de trois jours après l'accident, la victime est morte d'insuffisance cardiaque. Les principales leçons de ces accidents sont les suivantes.

- Des doses très élevées ne sont pas nécessairement garantes d'estimation par des techniques cytogénétiques. Comme dans les formes les plus sévères, l'évaluation dosimétrique est urgente et aiguë, il est indispensable de disposer de méthodes fiables et rapides pour estimer les doses au-dessus de $10 \mathrm{~Gy}$.

- La distribution de l'exposition est plus déterminante pour les décisions thérapeutiques que la dose moyenne telle qu'elle est évaluée classiquement. La greffe de moelle est indiquée uniquement dans les cas d'irradiation homogène 
et dans une gamme étroite de dose. Ceci souligne l'importance pour les médecins de s'appuyer sur les données physiques, telles que celles obtenues par la reconstitution de l'accident.

- La moelle osseuse ne peut pas être considérée comme un organe isolé, puisque l'irradiation induit des effets interdépendants multiples dont la chronologie d'apparition est conditionnée par les dynamiques cellulaires. La cascade des défaillances successives des différents systèmes et organes représente un des défis médicaux les plus difficiles à résoudre, auxquels certains spécialistes ont à faire face régulièrement. Au cours des décennies passées, cet aspect des grandes irradiations était occulté par le fait que les victimes ne dépassaient pas le cap de la grande aplasie.

- Bien qu'il soit difficile d'évaluer précisément le rôle des facteurs de croissance hématopoḯtiques dans la stimulation des cellules résiduelles de l'hôte, on peut conclure que les facteurs de croissance (GM-CSF et IL-3) contribuent à la prise rapide du greffon et à la maturation des cellules du donneur en cas de greffe (accident de Soreq) et à une restauration accélérée autonome (accident de Nesvizh).

\subsection{Gestion délicate}

La gestion de l'accident devient délicate dès que des difficultés d'ordre médical surviennent, en raison de la nature et de la gravité des lésions. Deux exemples récents sont fournis par un accident avec une source de gammagraphie et un accident de criticité, tous deux survenus en 1999.

À Yanango (Pérou, 20 février 1999), une source d'iridium 192 d'environ $1 \mathrm{TBq}$, perdue sur un chantier, a été ramenée au domicile d'un employé et ne fut retrouvée qu'au bout de 10 heures (IAEA, 2000a). Les doses aux tissus de la cuisse et du périnée furent estimées entre 10 et 30 Gy. La gravité des lésions de la cuisse a justifié son évacuation en France, où des traitements de pointe apportèrent une amélioration transitoire mais ne purent empêcher une rechute dramatique au bout de six mois, avec apparition de lésions des tissus profonds du bassin, que ni la clinique ni la reconstitution dosimétrique ne laissaient prévoir. Un an après l'accident, la victime était dans un état désespéré. À Tokaï-Mura (Japon, 30 septembre 1999), trois travailleurs d'une usine de fabrication du combustible sont victimes d'un accident de criticité lors d'une opération banale de dissolution d'oxyde d'uranium dans une solution concentrée de nitrate d'uranyle (IPSN, 1999). Vraisemblablement pour gagner du temps, la dissolution est effectuée directement dans la cuve de précipitation et décantation ; la masse d'uranium enrichi de $16 \mathrm{~kg}$ a largement dépassé la masse autorisée de $2 \mathrm{~kg}$ et la masse critique pour de l'uranium enrichi à $18,8 \%$ de $5,5 \mathrm{~kg}$. L'exposition mixte gamma neutron 
est dans le rapport 60/40 environ. Les soins médicaux ont été d'un niveau exceptionnel et ont fait appel aux techniques les plus modernes; il fallait faire face à une aplasie grave pour deux des patients, compliquée par des brûlures profondes et étendues. La victime la plus gravement atteinte (environ $10 \mathrm{~Gy}$ ) a reçu un facteur de croissance (G-CSF) et une allogreffe de cellules souches hématopoïétiques qui se sont traduits par des effets bénéfiques au plan hématologique. Elle est morte 83 jours après l'accident suite à des défaillances multiples (rénale, hépatique, cardiaque et pulmonaire). La deuxième victime (environ $5 \mathrm{~Gy}$ ) a reçu une greffe de cordon ombilical, d'efficacité limitée, puisque sa propre moelle s'est reconstituée en partie en quelques semaines (IAEA, 1999). Ces accidents permettent de tirer les leçons suivantes.

- Le facteur humain joue un rôle très important dans la genèse des accidents. Il est source d'erreurs graves de jugement, entraînant des prises de décisions lourdes de conséquences, toujours étonnantes chez des techniciens chevronnés.

- La reconstitution dosimétrique, quand elle peut reposer sur des informations sûres, apporte aux médecins des éléments d'appréciation irremplaçables; elle permet de prendre au bon moment les décisions opportunes, évitant ainsi au patient un grand lot de souffrances. Cependant, elle ne peut préjuger de l'évolution clinique, en particulier des rechutes à distance d'organes vitaux dont les lésions ont une évolution lente.

- L'efficacité des nouvelles thérapeutiques ne doit pas faire oublier qu'un irradié est un polytraumatisé et que les greffes de cellules hématopoüétiques, quelles qu'en soient l'origine et l'importance des effets secondaires, ont des indications très limitées dans les accidents d'irradiation.

- Les irradiations mixtes avec une composante neutronique importante provoquent des lésions particulières difficiles à gérer.

\subsection{Catastrophes}

L'exemple d'une catastrophe nucléaire civile est l'accident de Tchernobyl (Ukraine, 26 avril 1986). Il est particulièrement intéressant d'analyser cet accident sous l'angle de sa gestion médicale, à la lumière des leçons qui en ont déjà été tirées (Guskova et Gusev, 2001). Le bilan des effets aigus des rayonnements subis par le personnel de la centrale, les intervenants et les sauveteurs, a été de 28 morts. Quel que soit le type de traitement ( 13 transplantations de moelle et 6 de foie fotal ont été tentées, avec seulement deux survivants), les victimes qui souffraient d'aplasie médullaire compliquée par des brûlures sont mortes au cours du premier mois (Baranov et Guskova, 1990 ; Gale, 1987). Parmi la population, dans les régions particulièrement touchées par les retombées des premiers jours, un excès de cancers de la thyroïde de l'enfant est apparu en 1991. Le nombre de cancers imputables à l'accident s'élevait à plus de 800 en 1996 (Williams, 1996). 
Le nombre total de cancers de la thyrö̈de qui pourraient se révéler atteignait 10000 cas, mais il était impossible d'indiquer une valeur moyenne vraisemblable (Cardis, 1996 ; Nénot, 1996). En 1999, le nombre total de cancers de la thyroïde de l'enfant pour l'Ukraine, le Belarus et la Russie est estimé à environ 1500 , sans qu'il soit possible de prévoir si l'excès va continuer, se stabiliser ou décroître (Tronko et al., 1999 ; Ivanov et al., 1999).

- Les espoirs mis dans les transplantations de moelle osseuse ont été déçus. Parmi les raisons des échecs, il faut retenir que les expositions étaient hétérogènes et autorisaient l'espoir d'une régénération spontanée de la moelle. Les transplantations de moelle ont des indications limitées dans le traitement des irradiés accidentels.

- La gestion médicale d'un grand nombre de victimes est très délicate (237 diagnostics de syndrome aigu d'irradiation ont été posés à Tchernobyl). Un des premiers problèmes qui se pose est l'orientation des victimes vers des structures hospitalières adaptées. Il n'existe pas de solution miracle : sans une préparation minutieuse et des plans d'urgence prenant en compte des situations variées et vraisemblables, la situation ne sera pas maitrisable et aboutira à un fiasco.

- La même remarque s'applique à la protection de la population. Confiner la population, lui procurer dans des délais convenables de l'iode stable à titre prophylactique et mettre en auvre les moyens d'évacuation soulèvent des problèmes d'ordres logistique, social, psychologique et économique.

- L'excès de cancers de la thyrö̈de chez les enfants n'aurait pas soulevé de polémique si les moyens nécessaires à la mesure et au contrôle avaient été disponibles en temps voulu. Les ressources diagnostiques et thérapeutiques nécessaires pour traiter les malades sont considérables et requièrent un engagement poursuivi.

\section{Accidents non reconnus d'emblée}

Le nombre d'accidents dont l'origine radiologique est identifiée par hasard augmente avec le temps. C'est souvent l'état des victimes qui permet au médecin traitant d'évoquer la cause radiologique de l'accident. À ce propos, il est permis de s'interroger sur le nombre d'accidents graves méconnus, dont les conséquences sont attribuées à des causes banales.

\subsection{Nombre réduit de victimes}

Les accidents qui fournissent des exemples de cette situation sont très nombreux, qu'il s'agisse de sources industrielles ou de sources médicales. Les exemples présentés ci-dessous sont représentatifs en relatif des accidents du secteur industriel (sept accidents) et du secteur médical (un accident). 
Dans la ville de Mexico (21 mars 1962), une famille entière a été décimée par une source de cobalt 60, trouvée dans une décharge (Andrews, 1963 ; Martinez et al., 1964). Un enfant de 10 ans est mort en avril, suivi de sa mère en juillet, sans que l'agent causal ait été identifié. C'est seulement en août, après le décès d'une fillette de 3 ans, que l'agent causal a été identifié. Il y eut cependant un décès supplémentaire en octobre. Le chef de famille a dû sa survie au temps limité qu'il passait chez lui. À Brescia (Italie, 13 mai 1975), les symptômes présentés par le manœuvre d'une installation agricole d'irradiation de céréales après son intrusion dans la chambre d'irradiation, ont d'abord été attribués à l'effet toxique d'insecticides. Après admission à l'hôpital, son exposition à l'une des quatre sources $(500 \mathrm{TBq})$ est restée ignorée pendant plus de deux jours par les médecins. La victime a été transférée en France le quatrième jour, où elle est morte le treizième jour (Jammet et $a l ., 1979 \mathrm{a}$ ). La dose moyenne à la moelle a été évaluée à $12 \mathrm{~Gy}$, avec une distribution très hétérogène.

À Setif (Algérie, 5 mai 1978), le diagnostic de l'accident causé par une source d'iridium 192 de $925 \mathrm{GBq}$ perdue et récupérée par une famille n'a été effectué que 38 jours après le début de l'irradiation aiguë de tous les membres de la même famille (Jammet et al., 1979b ; Mettler et Nénot, 2001a). C'est un communiqué des autorités de santé, alertées à la suite de la perte de la source, qui a permis à un médecin de campagne d'évoquer l'étiologie des symptômes. Au total, l'accident a causé la mort d'une femme âgée de 47 ans, des aplasies prolongées graves chez quatre jeunes femmes entre 14 et 20 ans et des brûlures radiologiques multifocales chez deux enfants de 3 et 7 ans, qui furent tous traités en France. À Casablanca (Maroc, 19 mars 1984) les mêmes causes qu'en Algérie causèrent les mêmes effets : une source d'iridium 192 de $600 \mathrm{GBq}$ perdue le 19 mars n'a été retrouvée que le 25 juin, soit 80 jours plus tard (Mettler et Nénot, 2001 a ; Parmentier et al., 1990). L'accident fut découvert fortuitement, après le décès à l'hôpital de quatre jeunes frères et sœurs dans un tableau d'aplasie profonde, identique à celui qui avait causé la mort de leurs parents quelques jours avant. Cet accident a causé huit morts et plusieurs irradiés qui ont présenté des aplasies de gravité variable ; il n'est pas exclu que cet accident ait fait d'autres victimes.

À San Salvador (El Salvador, 5 février 1989), trois employés d'une installation industrielle de stérilisation (source de cobalt $60,0,66 \mathrm{PBq}$ ) ont été irradiés alors qu'ils tentaient de débloquer le porte-source (IAEA, 1990). L'irradiation n'a été reconnue qu'avec l'apparition d'une brûlure trois jours après l'accident. Deux semaines furent nécessaires pour que l'installation soit à nouveau sûre. Les doses furent évaluées à 8,4 et $3 \mathrm{~Gy}$, avec une répartition hétérogène ; chez le plus gravement atteint la dose en certains points dépassait $10 \mathrm{~Gy}$. Les trois victimes furent traitées par un facteur de croissance (GM-CSF), dont l'efficacité n'a pu être prouvée. Les suites ont été très graves pour deux des irradiés : chacun 
a dû être amputé d'une jambe ; six mois plus tard, ils ont subi, l'un l'amputation de l'autre jambe et l'autre une intervention thoracique, cause de sa mort.

Dans un hôpital de Saragosse (Espagne, 10 au 20 décembre 1990), 27 malades ont reçu des doses plus élevées que prévues en raison du mauvais fonctionnement de l'accélérateur linéaire (Esco et al., 1993). Un réparateur avait pallié le défaut de l'appareil en modifiant l'énergie des électrons. Les patients ont développé des lésions des poumons, de l'oropharynx et de la moelle, compliquées par des atteintes vasculaires et cutanées. Comme les malades victimes de ce dysfonctionnement étaient porteurs de tumeurs graves, il est difficile d'établir avec exactitude la part de responsabilité de l'accident dans le nombre de décès. On estime que treize malades ou plus sont morts des suites directes de cet accident.

À Forbach (France, 13 août 1991), les trois manutentionnaires d'un accélérateur linéaire utilisé pour dépolymériser du Téflon ont été irradiés lors de leurs intrusions répétées dans l'appareil, alors que la tension accélératrice était maintenue afin de gagner du temps (Chanteur, 1992). Le débit de dose de l'émission résiduelle était encore de quelques Gy par seconde. Les lésions cutanées furent attribuées à un coup de soleil ; après plusieurs jours et devant l'évolution sévère présentée par une des victimes l'origine radiologique des lésions fut reconnue. Ce patient dût subir des greffes de peau répétées qui nécessitèrent son hospitalisation pendant une année entière. Son état est demeuré précaire. À Hanoi (Vietnam, 17 novembre 1992), un ingénieur a été irradié gravement aux mains par le faisceau d'un accélérateur linéaire d'un institut de physique, alors qu'il positionnait un échantillon pour l'analyser (IAEA, 1996b). Bien que la victime ait eu la notion immédiate de son accident, l'origine de ses brûlures ne fut reconnue qu'après deux semaines, à cause de l'aggravation de son état clinique. Quatre mois plus tard, le patient fut transféré en France dans un état grave et amputé de la main droite et des deux derniers doigts de la main gauche.

À une dizaine de kilomètres au nord du Caire (Égypte, 5 mai 2000), une source d'iridium 192 d'environ 1,5 TBq, perdue dans le sable près du village de Mit Halfa par une entreprise de soudure, a été trouvée par un agriculteur de 61 ans (El Naggar, 1997). Neuf personnes de sa famille furent alors exposées pendant sept semaines (débit de dose de $150 \mathrm{mGy}$ à 1 mètre de distance). L'aplasie, la fièvre et les éruptions cutanées furent d'abord attribuées à une virose. Ce n'est qu'après la mort de l'agriculteur et de son fils de 9 ans (30 et 42 jours après le début de l'irradiation) que la relation de cause à effet fut évoquée. Les autres membres de la famille les plus irradiés furent la mère de famille, ses deux filles, son fils de 22 ans et une de leurs tantes, qui reçurent entre 3 et 4 Gy. L'exposition prolongée de 150 habitants du village fut de l'ordre de 25 à $150 \mathrm{mSv}$; des analyses 
biologiques furent pratiquées chez 400 personnes. Les intervenants, au nombre d'une centaine, reçurent des doses entre 15 et $100 \mathrm{mSv}$.

- Trop de petites sources sont égarées. Leur perte occasionne souvent des blessés graves, qui arrivent tardivement en milieu médical. Des améliorations portant sur la sûreté et la gestion de ces sources sont indispensables. L'absence ou l'insuffisance de réglementation constitue un paramètre d'aggravation.

- Dans les installations lourdes, on constate que les accidents surviennent par défaut de règles élémentaires de sûreté, par manque de formation du personnel et par non-respect des règlements et consignes, quand il en existe.

- Les accidents rapportés en milieu hospitalier concernent des surdosages. Il ne faudrait pas oublier que des sous-dosages, qui ne se traduisent pas par des effets immédiats, peuvent aussi être lourds de conséquences pour les malades. Il convient cependant de reconnaître que les accidents à l'hôpital sont rares, comparés au nombre d'appareils puissants, au nombre de malades traités et aux traitements effectués.

\subsection{Victimes en nombre important}

La probabilité pour que de nombreuses personnes soient exposées augmente avec la durée pendant laquelle la perte du contrôle de la source et/ou sa disparition restent ignorées. Ces circonstances se retrouvent lorsque des erreurs se produisent au cours d'irradiations médicales ou lorsque des sources, le plus souvent industrielles et militaires, sont égarées. Quatre accidents survenus dans des hôpitaux et trois cas de sources égarées constituent des exemples particulièrement démonstratifs.

Le premier accident sérieux en milieu médical a eu lieu à l'hôpital de Colombus (Ohio, USA) : la correction erronée de la période du cobalt 60 a causé entre 1974 et 1976 la surexposition de 426 malades, dont les doses ont été entre 15 et $45 \%$ supérieures aux doses prescrites, selon le moment du traitement (Cohen et al., 1995). Parmi les 183 malades qui étaient encore vivants un an après leur traitement, plus du tiers présentaient des complications graves du système nerveux central (encéphale et moelle) et du système digestif (oro-pharynx, colon, rectum). Un résultat inverse a été obtenu entre l'automne 1982 et l'hiver 1991 à l'hôpital de Stokes-Upon-Trent (Royaume-Uni) à cause d'une erreur de calcul dans les nouvelles procédures de traitement : 1045 malades ont reçu des doses inférieures à celles qui leur avaient été prescrites (Ash et Bates, 1994). Les conséquences de cette sous-exposition, de 5 à $35 \%$, ne pourront jamais être précisées. Le troisième accident est survenu aux États-Unis dans quatre hôpitaux différents; une erreur de programmation répétitive sur le même type d'appareil a causé à cinq reprises entre juin 1985 et janvier 1987 le surdosage de malades (Newman, 1990). 
Le dernier épisode s'est produit alors que les hôpitaux concernés avaient été avertis. Les conséquences ont été des brûlures graves, des myélites, des paralysies, complications dont certaines ont entraîné la mort des malades. Le quatrième accident a eu lieu dans un hôpital d'Indiana (Pennsylvanie, USA, 16 novembre 1992). Au cours d'un traitement anticancéreux par brachythérapie à fort débit, une malade de 82 ans a regagné son établissement de postcure sans avoir été débarrassée de la source d'iridium 192 de 160 GBq (US NRC, 1993). Quatre jours plus tard, une infirmière a jeté dans les déchets médicaux un cathéter qui contenait la source. Le lendemain la malade est morte, sans que ce décès soit attribué à l'irradiation. Au total plus de 90 personnes ont été exposées. L'alerte a été donnée par l'appareil de contrôle de la station de traitement des déchets. Les doses les plus élevées ont été reçues par les infirmières au niveau de leurs mains.

Un exemple de perte de source industrielle est fourni par l'accident de Xinzhou (province de Shenzi, Chine, 19 novembre 1992) : après vingt ans de fonctionnement, les sources de cobalt d'un irradiateur industriel furent stockées dans un puits lors de la fermeture de l'installation en 1980 (Wu De-Chang, 1993). Lors de l'ouverture d'un chantier de construction, un ouvrier a trouvé un objet métallique et l'a ramené chez lui. L'activité résiduelle de la source était de $400 \mathrm{GBq}$. La victime, son frère et son père sont morts quinze jours plus tard. Ces décès ont alors été attribués à une maladie infectieuse. Comme le malade était venu à l'hôpital avec la source, environ cent personnes ont été irradiées : le personnel, les visiteurs et les manutentionnaires qui transportaient la source jusqu'au lieu de stockage des déchets. Ce n'est qu'après un mois que la cause réelle a été évoquée. La source a été retrouvée plus de deux mois après son détournement.

Un accident grave a eu lieu près de Tallin (Estonie, 21 octobre 1994), après qu'une source de césium 137, vraisemblablement d'origine militaire, ait été dérobée dans le centre de stockage de Tammiku et soit restée dans une maison pendant 27 jours. Le décès rapide de l'acteur principal, âgé de 25 ans, est attribué à une toxémie d'origine traumatique. Il a fallu qu'un jeune garçon de 14 ans présente des brûlures et une aplasie pour que l'étiologie soit évoquée par le pédiatre. La source, dont l'activité n'a pu être précisée, a été récupérée dans des conditions précaires. En tout, sept personnes ont été exposées à des doses significatives, et cinq ont subi des dommages graves (IAEA, 1998a). Trois années plus tard, onze gardes-frontière du centre d'entraînement de Lilo (Géorgie), ont été exposés à des sources militaires de césium 137, dont onze ont pu être identifiées. L'irradiation a duré approximativement un an, de mi-1996 à avril 1997 et a été formellement reconnue en août 1997 (IAEA, 2000b ; Peter et al., 2001). Les quatre victimes les plus gravement atteintes, toutes avec des brûlures multi-focales, ont été traitées en France et sept autres en Allemagne. Certaines ont bénéficié, pour la première fois dans le cas de brûlures radiologiques, de greffes de peau artificielle, 
couronnées de succès. Le nombre de sources retrouvées sur le terrain permettent de penser que de nombreuses autres recrues ont pu être exposées.

- Ces accidents, individuellement très différents par leurs origines (milieu médical, stockage de source) et leurs conséquences, possèdent un point commun : des mesures de prévention basées sur le bon sens auraient évité la plupart des morts et des blessés graves.

- De plus en plus d'accidents sont causés par des sources militaires abandonnées lors du repli des armées. L'absence de consignes lors de la passation des pouvoirs et le désintérêt des responsables militaires confèrent à ces accidents un caractère particulièrement odieux.

\subsection{Conséquences graves pour la population et l'environnement}

Trois accidents très lourds de conséquences pour les victimes ont eu en plus de graves répercussions sur l'environnement et ont causé d'importants dommages socio-économiques. Ils ont eu la même cause : source de radiothérapie laissée à l'abandon, avec dissémination de produits radioactifs. Deux autres accidents, survenus dans des hôpitaux et touchant pour l'un plus de cent malades et pour l'autre plusieurs dizaines, ont constitués de véritables drames nationaux.

À Juarez (Mexique, 6 décembre 1983), le démantèlement sauvage d'une source de téléthérapie constituée de billes de cobalt $60(15,6 \mathrm{TBq})$ a été découvert par hasard lorsqu'un camion chargé de fers à béton a déclenché le 16 janvier 1984 les alarmes du centre de Los Alamos aux USA (Sec. de Energ., 1984). Ces fers provenaient d'une fonderie mexicaine qui avait traité les résidus récupérés de la tête de la source. Environ 4000 personnes ont été exposées. Parmi celles-ci, environ 800 ont reçu des doses supérieures à 50 mGy et huit entre 1 et 7 Gy. Aucun décès n'a été déploré, en raison de l'étalement de l'exposition dans le temps. À Goiânia (Brésil, 13 septembre 1987), la dispersion d'une source médicale de chlorure de césium $137(50,9 \mathrm{TBq})$ a eu des conséquences plus graves que celles de Juarez, en raison de la grande solubilité du produit. Les premiers signes du syndrome aigu d'irradiation apparus chez les ferrailleurs ont été attribués à une pathologie tropicale, et il s'est écoulé deux semaines avant que l'accident soit reconnu (IAEA, 1983). Parmi les vingt personnes hospitalisées d'urgence, dix avaient reçu des doses entre 3 et 7 Gy. Vingt-huit personnes ont présenté des brûlures graves. La plupart des patients avaient de surcroît des contaminations internes, dont certaines correspondaient à des doses engagées élevées. Quatre victimes décédèrent. Une première décontamination de l'environnement, qui a nécessité la destruction de maisons, n'a été achevée qu'après trois semaines ; la ville et ses environs n'ont été considérés assainis de façon acceptable qu'en mars 1988. L'impact économique fut très lourd, et l'économie de toute la région a été affectée. 
La surexposition de 114 malades, dont des enfants, dans un hôpital de San José (Costa Rica, entre le 26 août et le 27 septembre 1996) a été considérée comme une tragédie par tout le pays. Des erreurs d'étalonnage d'une source neuve de cobalt 60 dans une unité de radiothérapie ont eu pour résultat d'augmenter les doses prescrites de 50 à $60 \%$ (IAEA, 1998b). La surexposition a eu des conséquences très sévères, qui se sont ajoutées aux maux pour lesquels les patients étaient traités et parfois aggravées par les conditions limites de certains traitements (fractionnements insuffisants, champs excessifs, etc.). Cet accident a causé des effets dramatiques chez 4 malades (tétraplégie, paraplégie, démyélinisation de la moelle cervicale et dorsale, effets digestifs et cutanés graves), des effets marqués pouvant s'aggraver dans l'avenir chez 16 autres, des risques pour le futur chez 26 autres ; seuls 22 patients n'ont pas souffert de l'accident, le plus souvent parce que le traitement a été interrompu avant que le surdosage soit effectif. Le nombre exact de décès directement attribuables à l'accident est d'estimation difficile (IAEA, sous presse a). Parmi les 61 décès qui eurent lieu au cours des deux années suivantes, il est vraisemblable que treize furent dus à l'erreur d'étalonnage et que quatre développèrent des complications qui ont pu être les causes principales de la mort. Parmi les 51 patients encore en vie en octobre 1998, deux souffraient de complications catastrophiques et douze présentaient des séquelles marquées et invalidantes (Mettler et al., 2001b).

À Bangkok (Thailande) une source de radiothérapie de cobalt 60 , non utilisée depuis son achat en 1974, a été volée les 25 et 26 janvier 2000 dans un entrepôt, puis rapidement revendue. L'activité de la source était alors d'environ $16 \mathrm{TBq}$. Pendant près de trois semaines, les quatre voleurs, deux jeunes ferrailleurs, le patron d'une boutique d'occasions et six membres de sa famille furent exposés à de fortes doses, plus ou moins localisées. Dix d'entre eux furent hospitalisés pour hémorragies et brûlures graves (Suzuki, 2000). La relation de cause à effet fut établie très tardivement. Trois personnes, âgées de 18, 23 et 44 ans, décédèrent au cours du deuxième mois après le vol de la source. Les informations sur les autres blessés furent contradictoires; sans doute afin d'éviter une panique parmi la population, et devant le nombre très élevé de personnes pouvant avoir été exposées, les autorités thaïlandaises ont été particulièrement silencieuses. Il a été néanmoins établi qu'une dizaine de victimes nécessitèrent des soins intensifs et que certains durent subir des amputations. Quarantequatre personnes, dont 5 femmes enceintes, ont présenté des signes éventuellement attribuables à l'irradiation. Un contrôle hématologique a été pratiqué chez plus de mille personnes; 19 d'entre elles présentaient une leucopénie. Cet accident a été considéré comme une catastrophe nationale, et la manque de transparence des acteurs politiques a favorisé les informations les plus invraisemblables. 
Au Panama (août 2001) une erreur dans la détermination des doses délivrées aux patients d'un service de radiothérapie a causé 28 victimes, dont certaines gravement atteintes. L'origine de la surexposition a été due à une modification dans la façon d'introduire les données de base dans le logiciel de dosimétrie ; cette modification, non autorisée par le système, avait pour but la mise en place d'écrans supplémentaires, afin de réduire le champ d'irradiation. De ce fait les doses délivrées aux patients ont été beaucoup plus élevées que les doses prescrites ; dans certains cas, elles ont atteint jusqu'à $120 \mathrm{~Gy}$ à l'intestin. Alors qu'en décembre des signes anormaux auraient pu être interprétés comme signes de surexposition, ce n'est qu'en février 2001 que l'erreur a été suspectée et que les traitements ont été interrompus. En dépit de la longue période au cours de laquelle l'erreur s'est répétée, le nombre de victimes (IAEA, sous presse b) est relativement petit, en raison des types d'affection traités, cancers génitaux de la femme et cancers de la prostate. Sur les huit décès survenus au cours du premier semestre 2001, trois sont manifestement consécutifs à l'accident, deux lui sont peut-être attribuables et un n'est pas en relation; quant aux deux derniers, les données sont insuffisantes pour pouvoir en tirer la moindre conclusion. Les survivants présentent des séquelles graves des sphères digestive et urinaire. Ils ne sont pas l'abri de complications tardives graves, comme des occlusions intestinales, des nécroses du rectum ou du côlon.

L'étude des circonstances de ce type d'accidents montre qu'ils ont un potentiel de survenue inacceptable.

- Il existe des facteurs communs entre ces accidents catastrophiques et ceux résultants de la perte de petites sources industrielles, qui constituent de sérieux handicaps à la prévention : absence ou carence de la réglementation, absence de suivi des sources, formation et responsabilisation insuffisantes $d u$ personnel.

- Un autre facteur commun mérite d'être individualiser: en matière de traitement ou de suivi médical, les moyens disponibles sont insuffisants et/ou inadaptés.

- L'accident de Costa Rica souligne l'importance pour la prévention d'erreurs graves de l'assurance qualité en radiothérapie et de la formation du personnel. En cas d'accident de surdosage, il est essentiel de mettre en place un suivi rigoureux médical et psychologique des survivants pendant au moins cinq ans, en les regroupant par type de pathologie: ceci permet d'améliorer les traitements, de conforter les malades et d'en tirer un enseignement rare.

\section{Accidents tenus secrets}

Ces accidents appartiennent pour la plupart au domaine nucléaire militaire et illustrent la guerre froide. L'apaisement des relations entre l'Ouest et l'Est ne 
permet cependant pas d'affirmer que la transparence totale est devenue de mise pour les accidents, même les plus anciens. Leur révélation peut être à l'origine de problèmes politiques graves et leur description peut dévoiler des secrets militaires ou industriels. Les grandes puissances nucléaires n'ont peut-être pas fini de lever le voile sur tous les incidents et accidents de parcours qui ont jalonné l'escalade aux armements. Les exemples les plus représentatifs sont fournis par la gestion désastreuse d'installations du complexe militaire soviétique des années 50 et 60 , et par les pertes en mer d'engins nucléaires par les deux superpuissances.

À Tcheliabinsk (Oural, 29 septembre 1957), une très large étendue de territoires dans les monts Oural a été contaminée par un rejet accidentel de produits de fission, survenue dans une installation secrète datant de l'immédiat aprèsguerre (Nénot, 1994). Cet accident a été révélé en 1990 par un réfugié politique. En réalité, la population était déjà chroniquement exposée depuis le début des années 50 par les rejets considérables du complexe militaire, dont près de $40 \mathrm{EBq}$ dans les eaux de rivière. Vingt villages (7 500 habitants) ont été évacués de façon définitive. L'accident survenu en 1961 en Atlantique montre les conséquences que peut avoir la recherche du secret à tout prix : pour ne pas abandonner un sousmarin nucléaire et risquer de le voir récupérer par des armées rivales, les autorités soviétiques ont demandé à l'équipage de procéder à des réparations de fortune. Au cours de ces opérations, plusieurs membres de l'équipage ont reçu de très fortes doses, et huit en sont morts. Un autre accident en Baltique a causé quatre morts ou plus en 1968. Bien qu'elles ne répondent pas à la définition d'accident, il n'est pas possible de passer sous silence les pertes d'armes et de réacteurs nucléaires militaires, dont le nombre est considérable : accidents aériens, tirs ratés, réacteurs de navires coulés, réacteurs de satellites désintégrés lors de leur retour dans l'atmosphère. Du côté américain on décompte 7 pertes en mer d'armes nucléaires (1950 et 1965), 3 missiles abîmés en mer et 2 sous-marins perdus avec leurs réacteurs et 3 chutes de satellites (Mettler et Allen, 1990). Du côté soviétique, 7 naufrages de sous-marins porteurs d'armes (1968-1989) et 1 destroyer perdu en mer Noire (1974).

- Les leçons de ces accidents de causes et de conséquences variées sont évidentes : les impératifs militaires relèvent beaucoup plus du secret et du but recherché que de soucis de sûreté et de protection. Seul un changement radical des mentalités, en premier lieu des responsables politiques, souligné par un souci de transparence, permettrait aux installations militaires des différents pays et aux opérations afférentes de posséder des niveaux de sûreté comparables aux meilleures installations civiles.

- Il est évident que les connaissances sur ce genre d'accidents et sur leurs conséquences ne sont, encore aujourd'hui, que très fragmentaires et incomplètes. 


\section{Conclusions}

Les conclusions à tirer du retour d'expérience concernent d'une part le diagnostic de l'accident et de la maladie présentée par les victimes et d'autre part la gestion technique de l'accident et le traitement de l'accidenté.

Les cas où l'accident n'est reconnu que tardivement sont trop nombreux. Il est possible d'imaginer que des accidents comparables dans leurs circonstances de survenue aient pu passer inaperçus. Quel que soit le délai écoulé entre l'événement et sa découverte, le temps perdu est porteur de conséquences graves : accroissement du nombre de victimes, aggravation de leur état, difficultés de diagnostic et de traitement. La prévention est essentielle et doit porter à tous les niveaux :

- réglementations adaptées,

- formation du personnel, volonté de transparence.

Ces conditions qui relèvent du bon sens permettraient de réduire le nombre de sources perdues et de celles trop facilement accessibles à des personnes non averties. Les efforts doivent porter particulièrement sur le facteur humain : la plupart des accidents incluent dans leurs arbres d'événements causaux des défaillances humaines comportant des violations graves des règles élémentaires de sécurité, qu'il convient de prévenir et d'empêcher.

La gravité de la maladie radioinduite n'est pas évidente d'emblée sur les seuls signes médicaux. La dosimétrie physique n'a pas toujours la place qu'elle devrait occuper et elle a ses limites : résultats parfois incertains, reconstitutions tributaires de témoignages humains. La dosimétrie biologique constitue certainement le passage obligatoire pour l'estimation de la dose absorbée, mais présente d'autres écueils. Seule la combinaison de méthodes physiques et biologiques permet d'accéder aux deux paramètres essentiels au diagnostic et au pronostic : dose absorbée moyenne notamment au niveau de la moelle osseuse, et distribution de cette dose dans l'organisme et les organes.

La gestion d'un accident qui implique un grand nombre de victimes, réelles ou potentielles, exige des moyens considérables. Une bonne gestion implique la prise de conscience de la dimension du problème par les autorités, qui doivent être préparées à ce type de situation. L'expérience acquise montre que les aspects psychosociologiques peuvent prendre le pas sur les aspects médicaux et sanitaires. La communication est particulièrement délicate et doit s'adresser à de nombreux relais : autorités, experts en sûreté et en radiopathologie, victimes, population et médias. 


\section{TABLEAU II}

Traitements médicaux et causes de la mort dans quelques accidents mortels. Medical treatments and causes of death in selected lethal accidents.

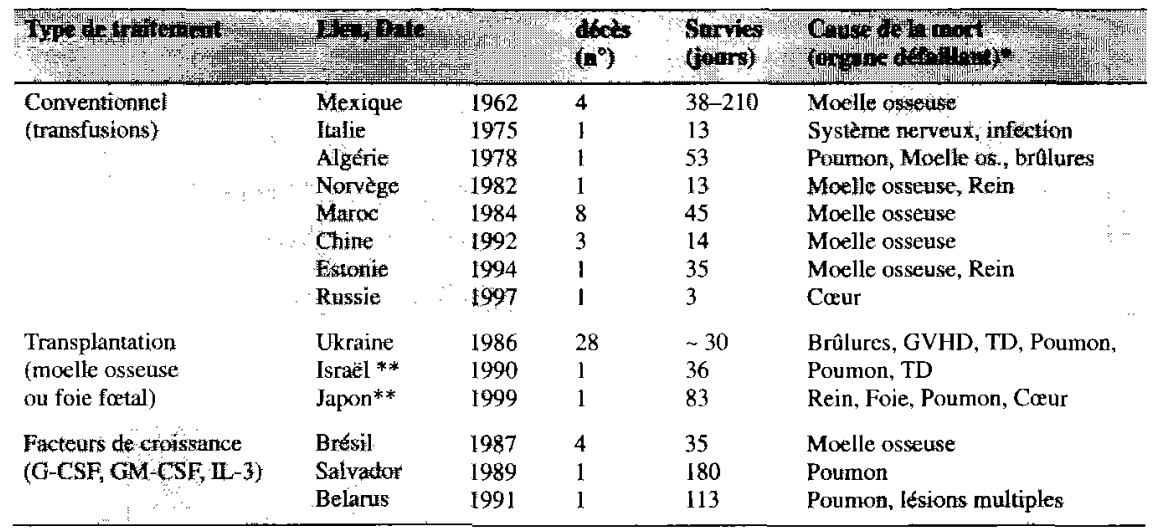

* TD : tube digestif ;

GVHD : Graft-Versus-Host-Discase ;

*** les victimes des accidents d'Israël et du Japon, qui apparaissent ici dans la case « transplantation » ont aussi reçu des facteurs de croissance (Israël : GM-CSF + IL-3, Japon : G-CSF).

* TD: gastro-intestinal tract;

GVHD: Graft-Versus-Host-Disease;

** the victims of the Israel and Japan accidents, who are mentioned in the "transplantation" category, received also growth factors (Israel: GM-CSF + IL-3, Japan: G-CSF).

La gestion médicale d'un irradié global est délicate et relève de nombreux spécialistes dont la coordination n'est pas toujours aisée. Le traitement des aplasies a bénéficié de progrès considérables depuis deux décennies. Les patients peuvent maintenant dépasser le stade de l'aplasie mais sont menacés par la défaillance d'autres organes et systèmes. Le tableau II montre que la mort des victimes survient le plus souvent à cause de la défaillance de plusieurs organes et systèmes. Seul le temps permettra de démontrer la réelle efficacité des facteurs de croissance hématopoïétiques. Il reste de nombreux progrès à faire pour maîtriser l'ensemble des syndromes induits par les rayonnements.

La gestion médicale des irradiations locales, dès que la couche basale de la peau reçoit des doses qui aboutissent à la nécrose des tissus et que la taille des brûlures devient importante, est extrêmement difficile. Force est de constater que peu de progrès ont été faits depuis plusieurs décennies. Cependant, l'application aux lésions radiologiques de techniques éprouvées dans d'autres domaines de la médecine autorise de sérieux espoirs, qu'il s'agisse du diagnostic ou du traitement. Une des victimes de l'accident de Géorgie, dont les trente-trois lésions constituent une illustration vivante des différentes formes cliniques des brûlures 


\section{J.-C. NÉNOT}

radiologiques, est aussi un exemple des progrès rendus possibles par les techniques modernes. Au plan du diagnostic et du pronostic, le bilan de l'extension des lésions a été possible grâce à la reconstitution dosimétrique par simulation numérique et surtout à l'imagerie par résonance magnétique, utilisée dans un tel contexte pour la première fois. Au plan du traitement, l'utilisation de peau artificielle a démontré ses avantages par rapport aux techniques classiques de greffes; ces dernières doivent être pratiquées tôt après l'irradiation afin d'éviter de redoutables surinfections, mais sont souvent rejetées et ne peuvent être répétées indéfiniment. La couverture par un derme artificiel recouvert d'une couche siliconée permet, sous le contrôle visuel direct, la reconstitution des tissus sous-jacents et du système capillaire, la colonisation du derme artificiel par les fibroblastes et la synthèse des protéines de la matrice intercellulaire. L'expérience est encore trop limitée pour affirmer que ces techniques constituent la voie d'avenir, mais il n'existe pas actuellement d'arguments contraires.

\section{RÉFÉRENCES}

Andrews G.A. (1963) Mexican Co 60 radiation accident, Isotopes Rad. Technol. 1 (2), $200-201$.

Ash D., Bates T. (1994) Report on the clinical effect of inadvertent underdosage in 1045 patients, Clin. Oncol. 6 (4), 214-226.

Baranov A.E., Guskova A.K. (1990) Acute radiation disease in Chernobyl accident victims. In: The medical basis for radiation accident preparedness 1 . Clinical experience and follow-up since 1979 (Ricks R.C., Fry S.A., Eds.), pp. 79-87, Elsevier/North Holland, New York.

Cardis E. (1996) Estimated long term health effects of the Chernobyl accident. In: One decade after Chernobyl. Summing up the consequences of the accident, pp. 241-279, IAEA, Vienna.

Chanteur J. (1992) Un accident d'irradiation, Médecins et rayonnements ionisants, $\mathrm{n}^{\circ} 3$, juillet 1992 , $5-6$.

Cohen L., Schultheiss T.E., Kennaugh R.C. (1995) A radiation overdose incident: initial data, Int. J. Radiat. Oncol. Biol. Phys. 33 (1), 217-224.

El Naggar A.M. (1997) Communication personnelle.

Esco R., Lopez P., Bellosta R., Baquedano J.E., Mateo P. (1993) Accidental overirradiation syndrome, Radiother. Oncol. 28, 177-178.

Gale R.P. ( 1987) Immediate medical consequences of nuclear accidents: lessons from Chernobyl, Jama $258(5), 625-628$.

Guskova A.K., Gusev I.A. (2001) Medical aspects of the accident at Chernobyl. In: Medical management of radiation accidents, 2nd edn. (Gusev I.A., Guskova A.K., Mettler F.A., Eds.), pp. 195-210, CRC Press, Boca Raton, London, New York, Washington DC.

IAEA (1983) The radiological accident in Goiania, IAEA, Vienna.

IAEA (1990) The radiological accident in San Salvador, IAEA, Vienna.

IAEA (1993) The radiological accident in Soreq, LAEA, Vienna.

IAEA (1996a) The radiological accident at the irradiation facility in Nesvizh, IAEA, Vienna.

IAEA (1996b) An electron accelerator accident in Hanoi, Vietnam, IAEA, Vienna.

IAEA (1998a) The radiological accident in Tammiku, Estonia, IAEA, Vienna. 
IAEA (1998b) Accidental overexposure of radiotherapy patients in San José, Costa Rica, IAEA, Vienna.

IAEA (1999) Report on the preliminary fact finding mission following the accident at the nuclear fuel processing facility in Tokaümura, Japan, IAEA, Vienna.

IAEA (2000a) The radiological accident in Yanago, IAEA, Vienna.

IAEA (2000b) The radiological accident in Lilo, IAEA, Vienna.

IAEA (2001) The criticality accident in Sarov, IAEA, Vienna.

IAEA (sous presse a) Follow-up mission on the accidental overexposure to radiotherapy patients in San José, Costa Rica, IAEA, Vienna.

IAEA (sous presse b) Investigation of an accidental overexposure of radiotherapy patients in Panama. IAEA, Vienna.

IPSN (1999) Fiche relative à l'accident survenu le 30 septembre 1999 à Tokaï-Mura (Japon), 14 octobre 1999, IPSN Ed.

Ivanov V.K., Gorsky A.I., Tsyb A.F. Maksyutov M.A., Rastopchin E.M. (1999) Dynamics of thyroid cancer incidence in Russia following the Chernobyl accident, J. Radiol. Prot. 19 (4), 305-318.

Jammet H., Gongora R., Le Gô, Doloy M.T. (1979a) Clinical and biological comparison of two acute accidental irradiations: Mol (1965) and Brescia (1975). In: The medical basis for radiation accident preparedness (Hübner K.F., Fry S.A., Eds.), pp. 9!-104, Elsevier/North Holland, New York.

Jammet H., Gongora R., Pouillard P., Le Gô, Parmentier N. (1979b) The 1978 Algerian accident: four cases of protracted whole-body irradiation. In: The medical basis for radiation accident preparedness (Hübner K.F., Fry S.A., Eds.), pp. I 13-129, Elsevier/North Holland, New York.

Martinez R.G., Cassab G.H., Ganem G.G., Gutman E.K., Lieberman M.L., Vater L.B., Linares M.M., Rodriguez H.M. (1964) Observations of the accidental exposure of a family to a source of cobalt-60, Rev. Med. Inst. Mex. Seg. Soc. 3 (supp. 1), 14-68.

Mettler F.A., Allen S.N. (1990) Military radiation accidents. In: Medical management of radiation accidents (Mettler F.A., Kelsy C.A., Ricks R.C., Eds.), pp. 45-88, CRC Press, Boca Raton.

Mettler F.A. Jr., Nénot J.C. (2001a) Accidental radiation injury from industrial source. In: Medical management of radiation accidents, 2nd edn. (Gusev I.A., Guskova A.K., Mettler F.A., Eds.), pp. 241-258, CRC Press, Boca Raton, London, New York, Washington DC.

Mettler F.A. Jr., Landberg T., Nénot J.C., Medina-Trejos F., Ching R., Garcia I., Perez-Ulloa V., Valerio-Hemandez M. (2001b) A 2-year follow-up of the radiotherapy accident in Costa Rica. In: Medical management of radiation accidents, 2nd edn., pp. 299-311, CRC Press, Boca Raton, London, New York, Washington DC.

Nénot J.C. (1994) L'Oural nucléaire. Bilan de 50 ans d'une gestion désastreuse. La revue du praticien, Paris, 44, 12, 1649-1654.

Nénot J.C. (1996) The Chernobyl accident: the consequences in perspective. In: One decade after Chernobyl. Summing up the consequences of the accident, pp. 475- 509, IAEA, Vienna.

Newman H.F. (1990) The malfunction "54" accelerator accidents 1985, 1986, 1987, 1990. In: The medical basis for radiation preparedness II. Clinical experience and follow-up since 1979 (Ricks R.C., Fry S.A., Eds.), pp. 165-171, Elsevier, New York.

Parmentier N., Nénot J.C., Parmentier C. (1990) Two cases of accidental protracted overexposure: aspect of an extensive bone marrow study. In: The medical basis for radiation accident preparedness II. Clinical experience and follow-up since 1979 (Ricks R.C., Fry S.A., Eds.), pp. 29-51, Elsevier, New York. 


\section{J.-C. NÉNOT}

Peter R.A., Carsin H., Cosset J.M., Clough C., Gourmelon P., Nénot J.C. (2001) Accident involving abandomned sources in Georgia, 1997. In: Medical management of radiation accidents, 2nd edn. (I.A. Gusev, A.K. Guskova, F.A. Mettler, Eds.), pp. 259-268, CRC Press, Boca Raton, London, New York, Washington DC.

Secretaria de Energia, Minas e Industria Paraestatal (1984) Accidente por contaminacion con cobalt60, Mexico 1984, CNSNS-IT-001.

Stavem P., Brogger A., Devik F., Flatby J., van der Hagen C.B., Henriksen T., Hoel P.S., Host H., Kett K., Petersen B. (1995) Lethal acute gamma radiation accident at Kjeller, Norway, report of a case, Acta Radiol. Oncol. 24 (Fasc. 1), 61-63.

Suzuki G. (2000) RERF dispatches radiation emergency specialists to Thailand at request of IAEA, RERF Update, 11, issue 1, Spring 2000, 4.

Tronko M.D., Bogdanova T.I., Komissarenko I.V., Epstein O.V., Oliynyk V., Kovalenko A, Likhtarev I.A., Kairo I., Peters S.B., Livolsi V.A. (1999) Thyroid carcinoma in children and adolescents in Ukraine, Cancer 86 (1), 149-156

US NRC, US Nuclear Regulatory Commission (1993) Loss of an iridium-192 source and therapy misadministration at Indiana regional Cancer Center, Indiana, Pennsylvania, on November 16 , 1992, NUREG-1480.

Williams E.D. (1996) Effects on the thyroid in populations exposed to radiation as a result of the Chernobyl accident. In: One decade after Chernobyl. Summing up the consequences of the accident, pp. 207-238, IAEA, Vienna.

Wu De-Chang (1993) Communication personnelle. 